Cahiers de recherches médiévales

\title{
Prudence et chevalerie dans le Livre des fais et bonnes meurs du sage roy Charles $V$
}

Susan J. Dudash

\section{(2) OpenEdition}

Journals

Édition électronique

URL : https://journals.openedition.org/crm/10872

DOI : $10.4000 / \mathrm{crm} .10872$

ISSN : 1955-2424

Éditeur

Honoré Champion

Édition imprimée

Date de publication : 10 décembre 2008

Pagination : 225-238

ISSN : $1272-9752$

Référence électronique

Susan J. Dudash, "Prudence et chevalerie dans le Livre des fais et bonnes meurs du sage roy

Charles V », Cahiers de recherches médiévales [En ligne], 16 | 2008, mis en ligne le 15 décembre 2011,

consulté le 15 décembre 2022. URL : http://journals.openedition.org/crm/10872 ; DOI : https://

doi.org/10.4000/crm. 10872 


\title{
酮RM
}

\section{Prudence et chevalerie dans le Livre des fais et bonnes meurs du sage roy Charles $V$}

\begin{abstract}
Celebrated for her defense of women, Christine de Pizan also exhibited a profound concern for her adoptive France and its successful governance through the proper education of its future sovereigns. This study will trace two of the essential components of a prince's instruction - prudence and chivalry - as they were redefined in her Livre des fais et bonnes meurs du sage roy Charles V.

Résumé : Célébrée pour sa défense des femmes, Christine de Pizan faisait aussi preuve d'un grand intérêt pour son pays adoptif et son bon gouvernement, qui pourrait être assuré par la formation appropriée de ses futurs souverains. Cette étude analysera deux des éléments essentiels de cet enseignement - la prudence et la chevalerie - tels qu'ils furent redéfinis dans son Livre des fais et bonnes meurs du sage roy Charles V.
\end{abstract}

\author{
Regardons le gouvernement \\ Des Rommains si victorieux : \\ Se vous lisez les glorieux \\ Fais de eulx, trouverés que savoir \\ Plus que force leur fit avoir \\ Les seigneuries qu'ilz acquistrent, \\ Car par le grant sens qu'ilz pourquistrent \\ Et orent ou gouvernement \\ Des batailles, qu'ilz prudemment \\ Menoient es particuliers \\ Cas de leurs fais, les chevaliers \\ Guerroians par sages cautelles \\ Avoient les victoires belles. \\ Et ou contenu de leurs fais \\ On les peut plus trouver parfais \\ De grant sens qu'en force de corps. [...] \\ Et ce que j'ay dit ci devant \\ De tuit li chevalier savant \\ Que l'en a trouvé et que on treuve, \\ Me doit en ce cas estre preuve \\ Que plus acquistrent par leur sens \\ Que par force, sicom je sens ${ }^{1}$
}

Je tiens à remercier B. Ribémont et N. Margolis dont les remarques pertinentes m'ont permis de perfectionner ce travail.

${ }^{1}$ Christine de Pizan, Le Livre du Chemin de long estude, éd. A. Tarnowski, Paris, Livre de Poche (Lettres gothiques), 2000, p. 380-82 (v. 4954-80). Ce passage introduit le portrait de Charles V par des considérations générales sur la Sagesse.

Cahiers de Recherches Médiévales, 16, 2008 
Célébrée pour sa défense des femmes, Christine de Pizan fut aussi un auteur politique de toute première importance. Auteur de traités d'éducation s'apparentant à de véritables miroirs de princes (et de princesses), elle montrait un grand souci de la prospérité de son pays adoptif, pensant en particulier qu'une formation et une éducation appropriées des futurs souverains de France étaient un important gage de réussite en la matière. Dans la tradition de ses premières œuvres didactiques écrites en vers comme son Epistre Othea de c. 1400 et son Livre du Chemin de long estude de 1402-1403 (œuvre dans laquelle elle faisait déjà l'éloge de la chevalerie savante $\mathrm{du}$ roi Charles $\mathrm{V}$ et cité ci-dessus en épigraphe), sa biographie officielle royale, le Livre des fais et bonnes meurs du sage roy Charles $V$ de 1404, marque son entrée dans le domaine de l'écriture politique en prose².

Tout comme elle le fera à nouveau dans ses miroirs plus théoriques, tel le Livre du corps de policie de 1406-1407, elle accorde une place centrale à l'éducation et à la formation du futur souverain dans sa biographie du sage roi. En effet, dans cette œuvre, les concepts de prudence et de chevalerie - essentiels à la formation de la tête du corps politique et du chef de l'armée - jouent un rôle de premier plan. Dans cette analyse, nous nous proposons donc d'examiner la manière dont Christine définit, voire redéfinit, la chevalerie à l'intention d'une nouvelle génération de monarques.

Plutôt que d'une chevalerie caractérisée par des décisions prises sans réflexion, des actions forcenées, et des attaques désorganisées et hâtives, dans le Charles V, il s'agit d'une 'chevalerie intellectuelle' fondée sur le «bon conseil», la réflexion, l'ordre, bref, sur la sagesse prudente en pensée, en parole, et en faits. Commandité par le duc de Bourgogne et destiné à l'édification du jeune dauphin (et, par extension, à la noblesse et à la France entière), cet ouvrage analyse la conduite chevaleresque idéale d'un futur roi français et tente de proposer des règles qui permettent aux règnes à venir d'être empreints de sagesse, de justice et de paix au service de tous les habitants du royaume.

Cette étude comporte trois parties, liées toutes les trois au concept de la prudence intellectuelle du roi modèle. Dans la première, nous considérerons les notions d'ordre et de modération, c'est-à-dire les mœurs prudentes et exemplaires du roi. Ensuite, nous examinerons une science militaire et un règne caractérisés par le «bon conseil » et la justice, la stratégie devant être fondée sur la diplomatie plutôt que sur l'usage de la force. Finalement, nous traiterons du rôle essentiel de la parole comme arme suprême dans l'arsenal du diplomate royal intellectuel, à une époque où la noblesse française se trouve à une espèce de 'carrefour chevaleresque' entre hauts faits et exercice de la prudence et de la sagesse, autrement dit de la « sapience » dont le prince doit être pour Christine le porteur privilégié.

\footnotetext{
${ }^{2}$ M. Zimmermann, « Mémoire - tradition - historiographie. Christine de Pizan et son Livre du sage Roy Charles V», The City of Scholars, dir. M. Zimmermann et D. De Rentiis, Berlin, de Gruyter, p. 158-73 (p. 163) ; S. Solente, dans son éd. Christine de Pisan. Le Livre des fais et bonnes meurs du sage roi Charles V, Paris, Champion, 1936, 2 vol. (t. 1, p. 5, n. 2). Toute citation du texte du Charles $V$ se réfère au tome et à la page de cette édition, ci-après : $L F B M$.
} 


\section{Vers une nouvelle chevalerie : prudence au Moyen Âge tardif}

Dans son étude sur le Charles $V$, E. Hicks note que d'après cette œuvre l'honneur, tel qu'il était conçu dans le passé, cède la place à la raison et à la prudence 3 . Daniel Poirion suggère qu'un nouvel idéal, ou plus exactement, «une révision des valeurs chevaleresques ", est en cours durant le Moyen Âge tardif, révision élaborée par les sages qui «prétendent définir la vraie noblesse en combinant vertus chrétiennes, valeurs rationnelles et qualités aristocratiques $\gg{ }^{4}$. Quant au contexte historique, si nous ne prenons en considération que les défaites désastreuses de Crécy (1346) et de Poitiers (1356) pendant le règne de Jean II le Bon, père de Charles V, il est évident que les gestes chevaleresques et l'approche militaire du passé n'aboutissent plus aux résultats attendus ou souhaités ${ }^{5}$. La politique de la deuxième moitié du $\mathrm{XIV}^{\mathrm{e}}$ siècle et du début du $\mathrm{XV}^{\mathrm{e}}$ siècle exige une nouvelle approche pour la diplomatie, une redéfinition de l'idée même d'exploit chevaleresque. Écrivain engagé, Christine a essayé de répondre aux crises de son temps en définissant un souverain idéal savant et prévoyant, qui disposât de l'érudition du passé et du sage conseil de son temps, afin de présenter un modèle politique exemplaire pour les générations à venir.

Euvre divisée en trois parties, le Charles $V$ traite de la noblesse de courage (cœur), de la chevalerie, et de la sagesse ${ }^{6}$. Comme nous le verrons, pour Christine, toutes ces qualités sont liées à la prudence. À l'exemple du sage roi qui consulte toujours ses conseillers avant de prendre une décision à propos de n'importe quelle entreprise sérieuse, Christine, en suivant les écrits des Anciens, définit la noblesse de courage par les actions suivantes : «tendre à haultes choses, amer bonnes meurs

\footnotetext{
${ }^{3}$ E. Hicks, «Excerpts and Originality: Authorial Purpose in the Fais et bonnes meurs », Christine de Pizan 2000: Studies on Christine de Pizan in honour of Angus J. Kennedy, dir. J. Campbell et N. Margolis, Amsterdam, Rodopi (Faux titres, 196), 2000, p. 221-31 et 331-34 (p. 231) ; voir aussi N. Margolis, «Christine de Pizan : The Poetess as Historian », Journal of the History of Ideas, 47, 1986, p. 361-75 (p. 372).

${ }^{4}$ Le Poète et le prince, Paris, PUF, 1965, réimpr. Genève, Slatkine, 1978, p. 597 et chap. 16. Voir aussi P. Contamine, Guerre, état et société à la fin du Moyen Âge, La Haye, Mouton, 1972, p. 232, K. Forhan, The Political Theory of Christine de Pizan, Aldershot, Ashgate, 2002, p. 143, et D. Delogu, «Reinventing the Ideal Sovereign in Christine de Pizan's Livre des fais et bonnes meurs du sage roy Charles V", Medievalia et Humanistica, n.s. 31, 2005, p. 48-50. Voir également l'ouvrage de J. Krynen, L'Empire du roi. Idées et croyances politique en France (XIII ${ }^{e}-X V^{e}$ siècle),Paris, Gallimard, 1993 ; sur la prudence et la sapience, p. 208-24). Sur le rôle d'Aristote dans les écrits de Christine, voir la note 11 ci-dessous.

${ }^{5}$ Voir S. Pagot, «Du bon usage de la compilation et du discours didactique : analyse du thème 'guerre et paix' chez Christine de Pizan », Une femme de lettres au Moyen Âge. Études autour de Christine de Pizan, dir. L. Dulac et B. Ribémont, Orléans, Paradigme (Medievalia, 16/Études christiniennes), 1995, p. 39-50 (p. 45-46). Le refus d'abandonner les méthodes démodées et inutiles du passé eut à peu près les mêmes effets catastrophiques à la veille du $X V^{\mathrm{e}}$ siècle. À titre d'exemple, la défaite des armées chrétiennes à Nicopolis en 1396, défaite qui marqua la dernière tentative sérieuse de la part des croisés occidentaux pour récupérer la Terre Sainte pour plus d'un siècle.

${ }^{6}$ Sur l'ordre du Charles V, voir E. Hicks, art. cit., p. 229.
} 
et conduire ses fais par prudence $»^{7}$. Mais la prévoyance se révèle également indispensable dans d'autres domaines politiques.

Tout en détaillant les qualités essentielles du bon stratège (c'est-à-dire, la bonne fortune, le bon sens, la diligence et la force) dans la deuxième partie du texte, Christine note que Charles $\mathrm{V}$ « introduit par l'administracion de raison et grant prudence en toutes choses [...] en tous les fais generaulx et particuliers de ses guerres » $(L F B M 1,117-18)$. Tandis qu'elle insiste toujours sur la qualité de «vray chevalereux» que possède le roi, Christine indique que ce fut à cause de «la prudence et valeur du souverain prince » qui « garde de son pais comme bon pastour de ses ouailles » que les Anglais n'osèrent plus mettre les pieds en France (1, 24243). Le pouvoir militaire ainsi que le renom du souverain proviennent alors, non pas de la gloire sur le champ de bataille, mais plutôt de sa prévoyance, impliquant une action plus spéculative et intellectuelle que celle des chefs militaires français du passé8.

Quant à la sagesse du roi, sujet de la troisième et dernière partie de l'œuvre, elle se définit, elle aussi, par cette qualité essentielle de circonspection et de précaution: «sagece, el quel terme ou seul mot peut estre compris sapience, science et prudence » $(L F B M 2,7)$. Au chapitre suivant (III, 2), Christine précise que la sagesse repose sur quatre piliers : d'une part la pratique rationnelle de l'art (dans la fabrication et la production des objets matériels) et de la prudence (ce qui gouverne les actions pures) et de l'autre, l'entendement (ce qui considère les causes premières) et la science et la « sapience» (le savoir des causes secondes) ${ }^{9}$. C'est alors que nous arrivons enfin à une vraie définition de la prudence dans ce livre, quand Christine s'appuie sur l'autorité de Cicéron («Tulle») : «comme prudence par memoire des choses passées porvoie aux futures, car [...] 'les parties d'elle sont mises: memoire, intelligence et pourveance' $»^{10}$. Tandis que le concept de la prudence reste complexe, ${ }^{11}$ Christine insiste, dans son texte consacré à la gloire du monarque, sur la «perfaitte prudence» du roi, de sa prévoyance en toute situation ${ }^{12}$.

\footnotetext{
${ }^{7}$ LFBM 1, 9-10.E. Hicks et Th. Moreau parlent de 'noblesse de courage' ou de 'caractère', Christine de Pizan. Le Livre des Faits et Bonnes Mours du roi Charles Vle Sage, tr. E. Hicks et Th. Moreau, Paris, Stock, 1997, p. 24, ci-après : ChV.

${ }^{8}$ Cf. l'analyse de N. Margolis, art. cit., p. 366, 372.

${ }^{9}$ LFBM 2, 10. Voir aussi M. Carruthers, The Book of Memory, Cambridge, Cambridge University Press, 1990, p. 66.

${ }^{10}$ LFBM 2, 21. Comme le démontre Solente, cette citation est tirée mot pour mot du commentaire de saint Thomas d'Aquin sur la Métaphysique d'Aristote, ibid., n. 2 : Mét., t. 24, p. 342 , col. 2 et t. 24 , p. 338, col. 2. Cf. K. Green, art. cit., p. 117, 119. Sur les sources littéraires de Christine, voir l'introduction de Solente, $L F B M$, xxxii-xlix, l'article d'A. Kennedy sur l'atrempance, à paraître dans les Actes du colloque international Christine de Pizan 2006, Paris, Champion, (Études christiniennes, 16), 2008, l'article de D. Delogu dans ce numéro et la note 11 ci-dessous.

11 Si l'étude détaillée de l'influence de la pensée aristotélicienne sur les œuvres contemporaines dépasse les limites de la présente analyse, il ne faut pas oublier que le sage roi chargea son ancien précepteur, Nicole Oresme, de nouvelles traductions et commentaires en langue vulgaire de l'Éthique à Nicomaque (achevé en 1370) et des Politiques (c. 1372). Cf. $L F B M 2,43$ et notes. En effet, la prudence forme le sujet de dix des dix-sept chapitres du livre
} 
La prudence, qui constitue un leitmotiv dans la biographie du sage roi, sera aussi l'une des vertus les plus chères à Christine, comme en témoignent ses autres écrits didactiques, théoriques et/ou pratiques en prose destinés à l'édification du jeune dauphin comme le Corps de policie, le Livre des fais d'armes et de chevallerie, et le Livre de Prudence ${ }^{13}$. Pour ne citer ici qu'un exemple, dans le Corps de policie, l'auteur précise que Prudence est avant tout la première des quatre vertus essentielles dirigées par la reine Felicité, suivie de Justice, Force [courage], et Atrempance, toutes les quatre destinées à aider le prince à bien diriger son pays dans la justice et la paix, et à lui assurer un long règne ${ }^{14}$. Selon Jules Michelet, Charles V fut « le héros de la patience et de la ruse », le premier roi « qui ait su préparer de loin un succès, le premier qui ait compris l'influence, lointaine et lente, mais dès lors

6 de l'Éthique au cours desquels nous apprenons, par exemple, qu'à la différence d'une vertu morale comme Tempérance, Prudence est une vertu intellectuelle « qui est architectonique et principal et qui ordene de tout. Et est verité que politique et prudence sont .i. meïme habit et different aucunement. » Aristote, Le livre de ethiques d'Aristote, éd. A. Menut, New York, Stechert, 1940, p. 339, n. 17 et 19 ; cf. p. 344-45. Sur Oresme et Charles V voir C. Sherman, Imagining Aristotle: Verbal and Visual Representation in Fourteenth-Century France, Berkeley, CA, University of California Press, 1995, parties 1 et 2 et S. Babbitt, Oresme's Livre de Politiques and the France of Charles V, Philadelphia, American Philosophical Society (Transactions of the American Philosophical Society 75, 1), 1985. Sur le rôle d'Aristote dans les écrits de Christine, voir, entre autres, les études de J. Krynen, L'empire du roi, Paris, Gallimard, 1993, p. 215, 217, K. Forhan, op. cit., S. Lefèvre, « Christine de Pizan et l'Aristote oresmien », Au champ des escriptures, dir. E. Hicks, et al., Paris, Champion, 2000, p. 231-250, et K. Green, "On Translating Christine as a Philosopher», Healing the Body Politic, dir. K. Green et C. Mews, Turnhout, Brepols (Disputatio, 7), 2005, p. 117-37. Se référer aussi la note 12 ci-dessous.

12 LFBM 2, 21. Sur la prudence chez Christine, voir aussi C. Reno, «Le Livre de Prudence/Livre de la Prod'hommie de l'homme: nouvelles perspectives », L. Dulac et B. Ribémont, op. cit., p. 25-37, J. Quillet, «Sagesse et pouvoir selon Le Livre des fais et bonnes meurs du sage roi Charles $V$ de Christine de Pizan », id., D'une cité à l'autre, Paris, Champion, 2001, p. 305-12, M. Richarz, «Prudence and Wisdom in Christine de Pizan's Livre des fais et bonnes meurs du sage roy Charles $V »$, K. Green et C. Mews, op. cit., p. 99116, K. Green, art. cit., et L. Dulac, "Rhétorique, critique et politique dans le Livre de Prudence de Christine de Pizan : quelques aperçus », Actes du colloque de Montpellier-mars 2008- « La Vertu de Prudence de Jean Gerson à Gassendi » (à paraître).

${ }^{13}$ Sur la catégorisation du Charles V, voir les commentaires de Solente, LFBM 1, xxvi-xxxii, Hicks, ChV, p. 24, et B. Collett, «The Three Mirrors of Christine de Pizan», K. Green et C. Mews, op. cit., p. 1-18 (p. 9-10). Noter aussi que les Fais d'armes développent le côté pratique de l'enseignement du prince quand on le compare avec des textes didactiques tels le Charles $V$. Des analyses détaillées des différents aspects de ce manuel militaire pratique font le sujet de nos recherches actuelles.

${ }^{14}$ Le Livre du corps de policie, éd. A. Kennedy, Paris, Champion (Études christiniennes, 1), 1998, p. 2, 1.2. Voir aussi ibid., p. xvii-xix et $i d$., art. cit., ainsi que S. Dudash, « Social Class, Vice, and Gluttony in Fifteenth-Century France » (à paraître). Voir aussi la note 12 ci-dessus. 
réelle, des livres sur les affaires $\rangle^{15}$. Mais ses qualités de prévoyance ne représentent qu'une des deux faces de cette médaille royale. Charles V fut aussi le parangon de la modération.

\section{La «mesure » d'un roi et de ses mours prudentes}

Étant donné les excès de la cour de Charles VI, le goût du plaisir chez le futur Charles VII et le déclin des mœurs chevaleresques au profit d'une vie raffinée et somptueuse $^{16}$, il n'est pas étonnant que Christine souligne les qualités exemplaires de la modération chez Charles V. Si «tout sens bien ordonné» doit pardonner les transgressions de la jeunesse et «ramener à discipline» une âme née pour régner mais en proie au flot des passions et aux «assaultz natureulx $»^{17}$, il reste du temps pour mieux former les futurs souverains.

Continuateur du pieux saint Louis, opposé aux païens des siècles passés, et le complétant $^{18}$, Charles V se présente dans les ouvres de Christine de Pizan comme un antidote intellectuel aux outrances et à la lâcheté de son temps. Dans le Charles $V$, le sage roi sert de modèle de bonne conduite, celle raisonnable et mesurée d'un prince qui se consacre au bien-être de son royaume. Il s'agit aussi ici, avant tout, d'une question d'ordre, de discipline, et de modération. Selon Christine, Dieu, l'origine de toute sagesse, est aussi le créateur de tout ordre terrestre et céleste (LFBM 1, 31-32).

En tant que son représentant sur terre, le roi doit être ordonné dans ses mœurs et dans tous ses gestes. Comme l'illustre la personne de Charles V, ces qualités se manifestent extérieurement par son physique proportionné et harmonieux (quoique affaibli par sa maladie), mais aussi, et ce qui est plus important, se révèlent de façon remarquable dans sa maîtrise de soi, c'est-à-dire, dans son maintien calme et constant. Pour citer Christine: "Chault, furieux en nul cas n'estoit trouvé, ains amoderé en tous ses fais, contenences et maintiens, touz telz qu'apartienent à rempli de sagece hault prince $\gg^{19}$. Comme nous le verrons, ce sang-froid est essentiel au chef suprême d'une armée afin qu'il puisse parvenir à l'heureuse résolution de toute question politique ou militaire. Autre sujet, sur lequel nous reviendrons plus tard, le fait que même sa diction et son argumentation sont « tant ordennée[s] et par si belle arrenge, sanz aucune superfluité de parole » $(1,49)$. Il en va de même dans la plupart des chapitres de la première partie du livre. Les chapitres 1,15 et 16 , par exemple, décrivent la vie que devait mener le roi «en belle ordenance» $(L F B M 1,39)$ ou «en

\footnotetext{
${ }^{15}$ Histoire de France, VI, chap. 1, Euvres complètes, éd. P. Viallaneix, Paris, Flammarion, t. 5, 1975, cité dans Hicks, $C h V$, p. 27.

${ }^{16}$ LFBM 1, 184-85. N.B. que dans cet exemple, la déchéance entraîne une invasion anglaise.

${ }^{17}$ LFBM 1, 28. Voir B. Ribémont, «Le regard de Christine de Pizan sur la jeunesse », Cahiers de Recherches Médiévales, 7, 2000, p. 255-60 (p. 256).

${ }^{18}$ Cf. $L F B M$ 1, 94-97 pour l'association entre ces deux rois dévots. Cf. L. Walters, "Constructing Reputations: Fama and Memory in Christine de Pizan's Charles $V$ and L'Advision Christine ", Fama: The Politics of Talk and Reputation in Medieval Europe, dir. T. Fenster et D. Smail, Ithaca, Cornell University Press, 2003, p. 118-42 (p. 131, 134).

${ }^{19}$ LFBM 1, 48-49. À ce propos, voir aussi L. Dulac, «La gestuelle chez Christine de Pizan : quelques apercus ", E. Hicks, et al., Au champ, op. cit., p. 609-26 (p. 619), et D. Lechat, «L'art du portrait dans Charles V», Contexts and Continuities, dir. A Kennedy, et al., Glasgow, University of Glasgow Press, 2002, t. 2, p. 515-29 (p. 519). Cf. A. Kennedy, art. cit.
} 
rigle de vie ordenée » $(1,43)$; le chapitre 20 célèbre la réglementation de la cour auprès de sa reine «Quel triomphe! Quelle paiz! En quel ordre, en quel coagulence regulée en toutes choses estoit gouvernée » $(1,53)$; le chapitre 21 est consacré à l'alimentation sobre du roi et à la discipline de ses enfants $(1,57 \mathrm{ff}$.), et par la suite, à son choix de vêtements honnêtes et «non trop curieuses en cost desordenné » et, enfin, à la sobriété et à la modération $(1,85)$. Ses dépenses et ses impôts sont, eux aussi, ordonnancés selon son rang et les besoins de l'État (1, 68-72, cf. 1, 79-82). Qualité essentielle chez le souverain qui fait face à des sujets rebelles (ainsi que dans tout comportement régalien), la réaction réfléchie et tempérée du roi exemplaire évite au royaume, précise Christine, des maux politiques encore plus graves et coûteux qui pourraient provenir d'actions inspirées par un désir spontané de vengeance $(1,66-67)$. C'est ici, dans la première partie de son traité, que la sagesse s'unit définitivement à la prudence "mere et conduisserresse des aultres vertus » et au concept d'un règne juste, fondé sur le bon jugement objectif et la conduite équilibrée de son chef en paroles et en pensées ${ }^{20}$. Avant tout, c'est la maîtrise de soi qui définit ici le roi prudent et exemplaire. Il est à noter que la «souffisance » et la modération des membres de la société française furent aussi un thème cher à un contemporain et ami en littérature de Christine: le poète-guerrier, émissaire, et confident du roi Charles VI de France, Eustache Deschamps ${ }^{21}$.

\section{La chevalerie ordonnée et avisée : prudence en faits}

Le sage roi démontre sa maîtrise de soi et sa modération non seulement dans ses actions quotidiennes, mais aussi dans son comportement chevaleresque, le sujet de la deuxième partie du livre ${ }^{22}$. Cette prévoyance se retrouve dans l'exemplarité de la justice mesurée de Charles V, ainsi que dans ses actes militaires et diplomatiques, l'ordonnance de ses armées et des chefs de guerre, et dans son entourage.

En effet, Christine précise que la prudence militaire de Charles V se manifeste même dans les origines de l'expression chevalerie. Il y a deux côtés à cette pratique : d'une part, la loi qui concerne la justice ordonnée du bien public et de l'autre, la chevalerie qui existe pour la défense et la protection de tous. Dans le passé, elle nous le dit, on appelait les chevaliers milites, ou «les meilleurs d'un millier $»^{23}$; Christine préfère employer le terme latin, car il implique des actions méritoires, aspect qui manquait à la plupart des chevaliers français de son époque (LFBM 1, 116). Non seulement l'auteur insiste sur le concept du mérite attaché au

${ }^{20}$ LFBM 1, 59 et ibid., chap. 23 et 29. Pour le contexte économique, voir J. Krynen, op. cit., p. 219-220.

${ }^{21}$ Voir les études de M. Lacassagne, «Rhétorique et politique de la 'médiocrité' chez Eustache Deschamps», Autour d'Eustache Deschamps, dir. D. Buschinger, Amiens, Université de Picardie/Centre d'Études Médiévales, 1999, p. 115-26 et "Eustache Deschamps: Discours et Société », thèse de doctorat, Washington University, 1994. Se référer aussi à A. Kennedy, art. cit. et S. Dudash, «Gluttony », art. cit.

${ }^{22}$ Cf. L. Dulac, "De l'art de la digression dans Le Livre du Charles $V$ du sage roy Charles V», Revue des langues romanes, 1, 1993, p. 115-26 (p. 120-22).

${ }^{23}$ L'étymologie peut être tirée d'Isidore de Séville : "Miles dictus, quia mille erant ante in numero uno », Etym., 9, 3, 32. Je remercie B. Ribémont pour cette référence. 
titre de chevalier, mais elle énumère aussi les hauts faits de Charles V comme « vray milite »- et avec lui, ceux de sa parenté royale et de ses proches, ainsi qu'on peut le voir dans la galerie de portraits royaux et militaires qui constituent la deuxième partie de la biographie ${ }^{24}$. Dans celle-ci, il ne s'agit pas simplement de dresser un panégyrique, mais d'analyser des faits exemplaires, ce qui est souligné par la métaphore de la chaîne (qui signale une certaine interdépendance et un équilibre entre les membres et les fonctions de la société chevaleresque) et se trouve résumé dans ce proverbe placé vers la fin de la deuxième partie: «Selon seigneur maignée duite $»^{25}$. Ce n'est pas la cérémonie seule qui définit l'état chevaleresque, mais plutôt «l'ordenence » c'est-à-dire des règles, la pratique et l'art des armes, et l'agencement nécessaire à toute équipe et à chaque mouvement militaires $(L F B M 1,119)$.

Comme preuve des actions chevaleresques du roi, qui sont fondées en doctrine, l'auteur met l'accent sur l'administration de la justice royale. Pour qu'elle soit équitable, voire juste, la justice doit être appliquée de façon raisonnable et logique à tout le monde, sans distinction de rang social ${ }^{26}$. Il est juste aussi que chaque état social participe, au moins en théorie, au Conseil ${ }^{27}$. La question de classe sociale mise à part, la justice bien mesurée est l'une des leçons les plus précieuses pour Christine et un thème auquel elle revient dans ses autres écrits politiques comme le Corps de policie, entre autres ${ }^{28}$.

Dans la deuxième partie de son Charles $V$, Christine consacre plusieurs chapitres à l'exposition de la justice "mesurée » du roi et offre de nombreuses anecdotes soulignant son importance pour le sage roi et pour bien public. Par exemple, on apprend que Charles $\mathrm{V}$ rend justice à une veuve dont la fille avait été violée par un serviteur de la cour; dans un autre exemple, il fit bénéficier un juif d'un jugement équitable (1, 61-62). Pour que la loi soit juste, la justice doit être rendue avec soin, surtout envers les faibles qui n'avaient pas d'autre recours, et à tous les membres de la société française, chrétiens ou non. Et qui plus est, dans son choix du meilleur candidat pour les postes officiels, le roi privilégie, nous assure Christine, un homme pauvre mais sage plutôt qu'un riche fou et désordonné29. Cet exemple témoigne de l'importance de bien ordonner l'administration et l'entourage du roi, et par extension, l'État français pour le bien de tous. En même temps, il enseigne au lecteur que la vraie justice est fondée sur une bonne décision, prise après mûre réflexion, c'est-à-dire, grâce à la prudence intellectuelle et juridique du

${ }^{24}$ LFBM 1, 117. Voir aussi, par exemple, LFBM chap. 11-17 (le premier sur les succès de Philippe le Hardi), 19-20, et 23-25 (sur les exploits de du Guesclin). Voir D. Lechat, art. cit., p. 517-19, 526-27 et C. Gauvard, «Christine de Pisan a-t-elle eu une pensée politique ?», Revue Historique, 250, 1973, p. 417-30 (principalement. 423-26).

${ }^{25}$ LFBM 1, 118-20, 130, et 243 respectivement. Dans ce dernier exemple, le proverbe se traduit « Tel maître, telle maison» (voir Hicks, ChV, p. 194). Cf. S. Dudash, «Christine de Pizan and the 'menu peuple' ", Speculum, 78, 2003, p. 778-831 (p. 823 et 825).

${ }^{26}$ Par exemple, $L F B M 1,60-61$.

${ }^{27}$ LFBM 2, 28-30. Cf. S. Dudash, « Pizan », art. cit.

${ }^{28}$ Ibid. Voir aussi K. Forhan, op. cit., p. 119-32 et M. Zimmermann, art. cit., p. 168

${ }^{29}$ LFBM 2, 60, C. Reno, "Christine de Pizan: At Best a Contradictory Figure ? », Politics, Gender, and Genre, dir. M. Brabant, Boulder, Westview Press, 1992, p. 171-91 (p. 175). 
roi. Appliquée avec soin et modération à tous et à toutes, la loi ne peut que fortifier le royaume contre ses ennemis.

L'autre aspect de la chevalerie que souligne Christine concerne la défense du royaume. Pour illustrer cette prudence guerrière, l'auteur met en valeur les succès militaires dans lesquels des tactiques bien conçues ont réussi à la noblesse française. Ramener un monde désordonné à l'ordre politique et social fait partie intégrante de ce devoir. Christine en énumère plusieurs exemples, y compris la gestion compliquée du problème des Grandes Compagnies par Charles $\mathrm{V}$ et la résolution des conflits en Flandres par son fils, Charles $\mathrm{VI}^{30}$. C'est aussi la leçon à tirer de la composition d'une armée et du déploiement de forces. Ce qui compte dans ce domaine est un mouvement militaire bien ordonné avec une prévoyance intelligente, et non pas l'ampleur, potentiellement chaotique, de forces nombreuses. Par exemple, Christine raconte la défaite de Xerxès, qui n'écouta pas le bon conseil d'éviter le combat, alors qu'il disposait de forces supérieures à celles de son ennemi ${ }^{31}$. À partir de ce cas, l'auteur montre l'importance de la capacité à envisager les résultats possibles et les difficultés potentielles que présente la conduite d'une aussi grande armée, comme en témoignaient les désastres français. En outre, cet exemple illustre pour le lecteur deux attitudes importantes adoptées par le sage roi : la nécessité de bien réfléchir avant de s'engager dans n'importe quel conflit militaire (sujet, nous le notons, du premier livre de ses Fais d'armes et de chevallerie), et celle de bien s'organiser et de bien user de ses forces dans toute situation diplomatique ${ }^{32}$.

Bien que se lancer directement dans les conflits militaires soit une attitude louée dans les chansons de geste et encore largement glorifiée chez des nobles du Moyen Âge tardif, l'auteur suggère qu'elle est imprudente, car on doit toujours envisager la fin et les résultats recherchés, et tenter d'éviter les coups de tête hasardeux. Sinon, de telles actions hâtives, inspirées par la passion du moment, qui conduisent à se jeter dans la mêlée sans réfléchir, peuvent entraîner des résultats néfastes $^{33}$. En citant ces exemples, Christine semble insister sur la nécessité de restaurer l'ordre social, peut-être parce qu'à l'époque où elle écrivait sa biographie du sage roi, la société française était privée d'un roi sain et raisonnable - Charles VI subissant ses premières crises de folie en 1392 - et se trouvait en proie au chaos du conflit avec les Anglais ${ }^{34}$. Mais quel rôle le roi doit-il jouer dans la guerre?

Un des éléments essentiels du bon agencement de l'armée concerne la question de la présence du roi sur le champ de bataille. Au lieu de se lancer directement dans le combat comme il l'avait fait pendant sa jeunesse et avant de devenir roi de France, Christine souligne avec soin que le roi Charles V préférait envoyer un expert bien choisi, un "prince de la chevalerie », pour le remplacer au

${ }^{30} L F B M 1,122-25$ et 1,163 . Voir aussi $L F B M 1,120-22$.

${ }^{31} L F B M 2,52$. Voir aussi $L F B M 1,201$.

${ }^{32}$ Par exemple, $L F B M 1,132-33 ; 1,215-16 ; 1,185$ et 2, 50-52 ; et 2, 21-30, respectivement.

${ }^{33}$ LFBM 2, 51-52 où Christine cite Charles V: "'En hastiveté ne gist pas la bonne ordenence ; quant nous verrons ceulz à qui parler en apertient, nous en ordenerons.' ».

${ }^{34}$ J. Krynen, op. cit., p. 200-01. 
$\operatorname{combat}^{35}$. Quoique l'auteur admette, dans la première partie de l'œuvre, que le roi souffrait d'une maladie qui affaiblissait son corps, elle ne fait aucune mention de son état physique dans le contexte militaire de ce chapitre (II, $3: L F B M 1,116-18$ ) ; tout au contraire, Christine insiste sur le fait que le roi devrait rester à l'écart du champ de bataille (sauf en cas d'extrême nécessité) afin d'éviter les maux qui retomberaient sur le royaume si son souverain était capturé pendant le combat ${ }^{36}$. Quant à la mort éventuelle du roi, elle pourrait entrainer la destruction de son peuple $(L F B M 1,115)$.

Ce sont des exemples à retenir, étant donné le sort désastreux du père de Charles V, Jean le Bon, qui, lors de la bataille de Poitiers en 1356, fut fait prisonnier par les Anglais et mourut hors de son pays, en Angleterre. Mieux vaut avoir un chef d'État dirigeant l'action hors de la mêlée que de le voir tomber éventuellement aux mains de l'ennemi. Au lieu d'une question de capacité physique, il s'agit plutôt dans ce chapitre (I, $3: L F B M 1,9-10)$ d'une manière de se protéger contre les revers de Fortune, et ce précepte est formulé d'après une bonne connaissance des leçons de l'expérience du passé et avec un regard vers l'avenir. Plutôt que d'y voir une marque de la faiblesse physique de Charles V, ou un manque de fortitudo royale, Christine note que cette démarche témoigne de la prudente conduite militaire du roi.

En effet, ce n'est que dans quelques chapitres de la partie II de sa biographie que Christine associe la jeunesse du futur roi Charles V, ses exploits chevaleresques, et sa maladie. Devançant certaines objections possibles, Christine répond ainsi : «que par recreandise n'alast en personne aux armées de ses guerres, n'estoit mie, car, ou temps qu'il estoit duc de Normandie, ains son couronnement, avec son pere, le roy Jehan, maintes fois y ala, et aussi, lui seul chevetaine de grans routes de gens d'armes » $(1,131)$. Essentiel à son argumentation est le fait que le roi participait au combat avant son couronnement et ce n'est qu'après son sacre qu'il commença à se tenir à l'écart pour mieux planifier ses stratégies. Remarquons aussi que Christine indique qu'il souffrait depuis son couronnement d'une maladie invalidante $(1,131$ 32). Ce qui compte pour l'auteur — et ce qui détermine la vraie valeur du roi-, c'est sa capacité intellectuelle à bien régner et à bien diriger de façon ordonnée et raisonnable ses sujets, et non pas sa force physique et personnelle. Par exemple, Christine insiste sur la supériorité de la stratégie par rapport au combat: que celui qui «sanz cesser ouvroit en toute peine pour la publique utilité, doie estre reprimé, n'est mie raison, car dit Vegece que plus doit estre louée chevalerie menée à cause de sens que celle, qui est conduitte par effett d'armes $»^{37}$. Il en fut ainsi pour les

${ }^{35}$ LFBM 1, 115. Se référer aussi aux p. 120-22, où Christine explique comment le roi Charles V peut être un «vray chevalereux » même s'il avait passé la plupart de son temps à l'abri de ses châteaux.

${ }^{36}$ LFBM 1, 115. Pour le portrait physique du roi: 1, 48-49; voir C. Sherman, «Representations of Charles V of France (1338-1380) as a Wise Ruler», Medievalia et Humanistica, n. s. 2, 1971, p. 83-96 et id., The Portraits of Charles V of France, New-York, New York University Press/College Art, 1969.

${ }^{37}$ LFBM 1, 132-33. Cf. Fais d'armes: «Chaton le vaillant combatant, par laquel force et vertu d'armes orent Rommains maintes belles vittoires et qui oncques en bataille ne fu desconfit, dit que plus cuidoit avoir prouffite a la chose publique en l'escripture des regles, enseignemens et discipline d'armes, que il avoit composé et en fait livres, que en chose qui il oncques fust faitte de son corps. » éd. C. Laennec, «Christine antygrafe: Authorship and the 
Romains qui «plus acqueissent seigneuries et terres par leur sens que par force» comme « le fist nostre roy », c'est-à-dire, Charles V $(L F B M 1,133)$. Dans ce même passage, Christine énumère ensuite les actions du sage roi en comparant ses œuvres à celles de Charlemagne. C'est alors qu'elle peut conclure que Charles V est « digne d'avoir le nom et tiltre de perfaitte chevalerie » (ibid.).

\section{Le pouvoir de la parole : prudence en dits}

Ce n'est pas par hasard que Christine revient à plusieurs reprises sur le langage et l'expression de Charles V. La parole fonctionne comme l'une de ses armes les plus précieuses et les plus efficaces, et concerne à la fois l'oral et l'écrit. Tout au long du Charles V, Christine souligne la qualité supérieure de l'éloquence, de la diction, et de la façon de raisonner du roi. Dans la première partie du livre, par exemple, nous trouvons un tel éloge : «sa belle parleure tant ordennée et par si belle arrenge, sanz aucune superfluité de parole, ne croy que rethoricien quelconques en lengue françoise sceust riens amender. » $(L F B M 1,49) .{ }^{38}$ Néanmoins, sa maîtrise de l'art de la rhétorique - habilité pour laquelle il était célèbre au-delà des frontières de la France, comme «advocat» selon les Anglais $^{39}$ - joue un rôle de première importance dans la troisième partie de l'œuvre, à propos de la résolution des conflits diplomatiques les plus graves, comme nous le verrons ${ }^{40}$.

Pour mieux appréhender l'écrit et s'informer, Charles V se vouait à l'étude, commanditait de nouvelles traductions en langue vulgaire des textes anciens et de ceux des savants contemporains, et apportait son soutien à l'Université de Paris, l'un des sièges les plus importants du discours intellectue ${ }^{41}$. De tous les sept arts libéraux, Christine met l'accent sur son étude de l'astrologie comme le sommet des études théoriques, avec la rhétorique ( $L F B M 2,15-19$ et 33-36 $)^{42}$. De plus, Christine illustre cette habilité en incluant dans son texte les réponses royales écrites à la troisième ainsi qu'à la première personne $(2,57-62)$, enregistrant les propres mots du roi dans des chapitres consacrés à ces citations mémorables $(2: 49-52,57-62,68$ -

Self in the Prose Works of Christine de Pizan, with an Edition of B.N. Ms. 603 Le Livre des fais d'armes et de chevallerie ", thèse de doctorat, Yale University, 1988, t. 2, p. 23. Cf. The Book of Deeds of Arms and of Chivalry, éd. C. Willard et tr. S. Willard, University Park, Penn State Press, 1999, p. 13-14 (traduction basée sur le MS Bruxelles BR 10476).

${ }^{38} \mathrm{Cf}$. LFBM, 2, 75-76. À part ses propres dons rhétoriques, Charles V s'appuyait sur le conseil des sages et des experts avec lesquels il pouvait discuter de la bonne voie à suivre dans n'importe quelle situation sérieuse ( $L F B M 1,125)$.

${ }^{39}$ LFBM 2, 80. Même Pétrarque a noté l'éloquence du roi sage ; voir R. Delachenal, Histoire de Charles V, Paris, Picard, 1909, t. 2, p. 363 et C. Sherman, art. cit., p. 85.

${ }^{40}$ Sur la sagesse et la parole « souveraine », voir L. Dulac, «Unité et variations de la sagesse dans le Livre des fais et bonnes meurs du sage roy Charles V", Revue des langues romanes, 42, 1988, p. 307-15 (p. 313). Cf. B. Ribémont, art. cit., p. 259.

${ }^{41}$ C. Sherman, art. cit., p. 90-91 et $L F B M 2,11-13$. Voir aussi la note 11, ci-dessus.

${ }^{42}$ Voir à ce sujet B. Ribémont, "Christine de Pizan, Isidore de Séville et l'astrologie : compilation et 'mutacion' d'un discours sur les arts libéraux ", à paraître dans Christine de Pizan 2006, op. cit. et $i d$., "Christine de Pizan et les arts libéraux : un modèle à géométrie variable », à paraître dans French Studies. 
69, 79-81), et rapportant ses bons mots ainsi que ses dernières paroles, prononcées sur son lit de mort $(2,184-92)$, pour ne citer que quelques exemples.

Faisant le pont entre l'oral et l'écrit, le roi témoignait de son érudition dans la résolution diplomatique des conflits, des plus minimes aux plus grands. Voilà le thème central de la troisième partie de la biographie : la capacité du roi à résoudre les conflits les plus sérieux, d'une portée internationale et religieuse, grâce à son intelligence prudente et bien informée, et à sa parole juste. Christine montre son habileté tout au long des douzaines de chapitres du Charles $V$, la prudence de sa parole prenant la première place dans deux cas particuliers : la visite en France de l'empereur Charles IV (1378) et la question du Grand Schisme de l'Église catholique (1378-1417).

Charles $\mathrm{V}$ fait preuve de diplomatie pendant toute la durée de la visite impériale ${ }^{43}$, mais, pour ce qui nous concerne ici, c'est plutôt à la fin de cet épisode, au moment où il plaide sa cause dans le conflit qui l'oppose aux Anglais devant le juge suprême temporel, l'empereur, qu'il déploie au mieux ses talents ${ }^{44}$. Dès le début du récit, l'auteur met l'accent sur «le sage roy, qui en son entendement avoit science et rethorique souveraine en lenguage » (LFBM 2, 116). Qui plus est, Christine souligne le fait que, avant d'entrer en négociation avec l'empereur, le roi français avait déjà épuisé toutes les autres possibilités diplomatiques pour résoudre les problèmes provoqués par le fait que les Anglais avaient violé les termes de leur traité avec les Français $(2,118-20)$. À titre d'exemple, dans ce même passage, Charles V avait envoyé des émissaires au roi anglais comme représentants de sa personne; dans la sphère diplomatique, la fonction de ces émissaires constituait le complément rhétorique et politique des chefs de guerre sur le champ de bataille. Préférant d'autres méthodes de résolution du conflit que la guerre ouverte, Charles $\mathrm{V}$ demande le soutien de l'empereur - et l'obtient -, ce qui représente le triomphe de son aptitude à la diplomatie servie par l'art de la rhétorique (2, 120-21).

Le deuxième grand exemple des tentatives rhétoriques du roi pour résoudre un grave conflit est celui de l'élection des papes, sujet auquel l'auteur consacre plusieurs chapitres de la troisième partie du livre. Christine insiste, à plusieurs reprises, sur le fait que le roi avait mené cette affaire avec le plus grand soin possible. Au concile de Paris en 1378, il s'entoura de théologiens, de clercs, de prélats, et de chevaliers pour discuter de tous les aspects de la question: s'il fallait soutenir Clément VII ou Urbain $\mathrm{VI}^{45}$. Une fois son jugement arrêté, il envoya des émissaires aux autres souverains européens, mais le conflit éclata avec force ( $L F B M$ $2,148-50)$. Même bien renseigné comme il l'était, le roi ne pouvait prévoir toutes les conséquences, mais quand il se rendit compte qu'il fallait rouvrir la discussion, il commença à planifier un concile, au cours duquel tous les monarques purent

\footnotetext{
${ }^{43}$ Voir, sur cette entrevue, F. Autrand, «Mémoire et cérémonial : la visite de l'empereur Charles IV à Paris en 1378 d'après les Grandes Chroniques de France et Christine de Pizan », L. Dulac et B. Ribémont, op. cit., p. 91-103.

${ }^{44}$ Voir M. Richarz, art. cit., p. 110.

${ }^{45}$ LFBM 2, 147. Voir R. Blumenfeld-Kosinski, Poets, Saints, and Visionaries of the Great Schism, 1378-1417, University Park, Penn State Press, 2006, p. 156-58.
} 
exprimer leur point de vue et trouver ensemble une solution ${ }^{46}$.Comme l'indique Christine $(2,158$, le sage roi mourut avant que l'on pût se réunir. Le fait qu'il faisait de son mieux pour employer tous ses dons rhétoriques et diplomatiques ainsi que sa prudence comme un vrai chevalier (du Christ) est bien illustré par ce dernier récit. Même dans les revers ou la défaite, Charles V présentait l'exemple d'un souverain parfaitement sage, voué au bien de son peuple, français ou chrétien.

\section{Conclusion}

Avec son Charles $V$, Christine ouvre un discours sur une nouvelle conception de la chevalerie fondée sur la sagesse et la prudence en mœurs, actions et paroles. Comme nous avions commencé par une citation du Chemin, revenons à ce texte et aux propos de Christine à la conclusion de son portrait de Charles V :

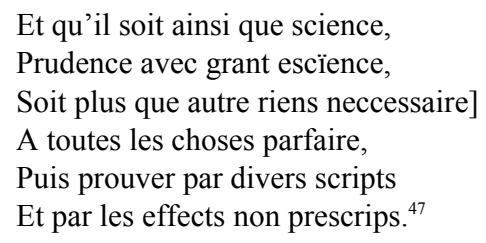

S'expriment ici non seulement l'importance de la prudence et de la sagesse, mais aussi la portée de la parole. De fait, dans la biographie christinienne, le discours prudent occupe une place essentielle: dans la première partie, où Christine «euvre [s]es levres », dans la deuxième, où elle met l'accent sur l'écrit, et enfin dans la troisième, qui réunit les deux aspects du savoir prudent ${ }^{48}$.

Il est certain que le destin de Christine est directement lié à celui de la France et, par extension, à celui du sage roi $^{49}$. À l'instar de cette biographie royale, Christine se mire dans l'image exemplaire de Charles V, le roi lettré auquel les écrits édifiants étaient si chers et qui fut, dans un sens, comme les érudits du passé, un des «ediffieurs en meurs redevables $»^{50}$. Christine, «douée de don [...] en amour d'estude » $(1,5)$, fut aussi un " architecte » de mœurs, faits, et dits. Au début de la troisième partie de son œuvre et en s'adressant au roi, l'auteur annonce : « me prent appetit de parler en sa personne» $(2,8)$. Mais cette fois-ci, elle parle aussi de la

${ }^{46}$ LFBM 2, 157-58. Voir M. Richarz, art. cit., p. 115-16 et R. Blumenfeld-Kosinski, "Christine de Pizan and the Political Life in Late Medieval France», Christine de Pizan : A Casebook, dir. B. Altmann et D. McGrady, New York, Routledge, 2003, p. 9-24 (p. 16).

${ }^{47}$ Chemin de longue étude, éd. A. Tarnowski, op. cit., p. 388, v. 5073-5078.

${ }^{48}$ Par exemple, $L F B M 1,4 ; 1,189-92$; et 2, 33-50.

${ }^{49}$ N. Margolis, art. cit., p. 372.

${ }^{50}$ LFBM 1, 5. Sur le lien entre l'auteur et le souverain, voir L. Dulac, «Digression », art. cit. Sur Christine comme architecte, voir aussi E. Hicks, art. cit., p. 225, D. Lechat, art. cit., p. 527 et 529, et L. Walters, «Fama», art. cit., p. 136-37. Cf. J. Holderness, "Castles in the Air? The Prince as Conceptual Artist», K. Green et C. Mews, op. cit., . 161-75 (p. 169-71). 
portée de la prudence - exemplifiée par le sage roi et inscrite dans les livres - pour les siècles à venir $(2,131-32)^{51}$.

Comme les Anciens, Christine a créé une œuvre qui ne pouvait être détruite ni par le feu ni par le fer, et qui contient des leçons à retenir, des témoignages de la conduite et du savoir prudents qui devaient être recopiés, répandus, et chantés partout (LFBM 2,131-32) - afin de prévenir l'effacement de ses récits des bonnes actions d'un roi exemplaire. Tout comme elle fut nourrie des miettes de la table de son père, c'est-à-dire de son érudition, Christine dut au sage roi d'être «nourrie de son pain » $(2,193)$.

Susan J. Dudash Fordham University/USA

${ }^{51}$ Voir aussi L. Walters, «The Royal Vernacular: Poet and Patron in Christine de Pizan's Charles V and the Sept psaumes allégorisés ", The Vernacular Spirit, dir. R. BlumenfeldKosinski, et al., New York, Palgrave, 2002, p. 145-82 (p. 151), id., «Fama», art. cit., p. 13132 et 136, id., "Christine de Pizan, France's Memorialist : Persona, Performance, Memory », Journal of European Studies, 35, 2005, p. 29-45, et M. Zimmermann, art. cit., p. 158 et 160. Cf. Fais d'armes, Laennec, éd. cit., p. 23. 